\title{
Nonconjugate Adaptation of Human Saccades to Anisometropic Spectacles: Meridian-
} specificity

\author{
H. G. LEMIJ,* H. COLLEWIJN*† \\ Received 4 April 1991; in revised form 12 August 1991
}

\begin{abstract}
Recently it has been demonstrated that saccades become different in size in the two eyes if a subject is adapted to anisometropic spectacles, which provide visual images of different magnitude to the two eyes. These nonconjugate adaptations adequately meet the requirements of those spectacles and, once acquired, they persist (with some reduction) even during monocular viewing. We now demonstrate that such nonconjugate adaptations of saccades can be meridian-specific, if there is a pressure for such meridian-specificity. This pressure was provided by means of a cylindrical spectacle-lens. Adaptations along a vertical, horizontal or oblique meridian did not transfer to the orthogonal meridian. These results demonstrate a capability of saccadic adaptation to deal with calibration problems restricted not only to one eye, but even to one specific plane of muscular action. Our results also suggest that the meridian-specific adaptations of oblique saccades take place at a stage before the decomposition of motor commands into separate horizontal and vertical components. The meridian-specific nonconjugacies were also expressed in smooth-pursuit eye movements. Post-saccadic drift adapted only along the horizontal meridian.
\end{abstract}

Hering's law Saccades Adaptation Aniseikonia Anisometropia Anisometropic spectacles Postsaccadic drift Smooth-pursuit

\section{INTRODUCTION}

Saccades are the rapid eye movements that shift our gaze from one visual target to another. In the absence of stimuli for vergence, the two eyes always make saccades of virtually the same size (for recent measurements see Collewijn, Erkelens \& Steinman, 1988a, b; Lemij, 1990,1991 a). This conjugacy is frequently alluded to as Hering's law of equal innervation (Hering, 1868). Normally, such yoking would be desirable as a default function, to prevent diplopia. Nonconjugate horizontal saccades can, however, readily be made in response to nonisovergent stimuli, i.e. during gaze shifts between targets differing in both distance and direction (Kenyon, Ciuffreda \& Stark, 1980; Enright, 1984; Erkelens, Steinman \& Collewijn, 1989). These nonconjugacies originate as a direct response to the target configuration and do not involve any permanent change in binocular motor programming. Such permanent changes are, however, possible as well. It has been recently demonstrated that nonconjugate saccades can be acquired as a default condition when subjects adapt to anisometropic

\footnotetext{
- Department of Physiology I, Faculty of Medicine, Erasmus University Rotterdam, P.O. Box 1738,3000 DR Rotterdam, The Netherlands.

†To whom all correspondence should be addressed.
}

spectacles (Erkelens, Collewijn \& Steinman, 1989; Zee \& Levi, 1989; Lemij, 1990; Schor, Gleason \& Horner, 1990; Lemij \& Collewijn, 1991a, b; Oohira, Zee \& Guyton, 1991). Anisometropic spectacles have lenses of unequal refractive powers, and therefore supply visual images of different magnitude to the two eyes. After adaptation, the eye provided with the smaller visual image made appropriately smaller saccades than its fellow eye. This adaptive nonconjugacy persisted (with some reduction) with one eye covered. This showed that the change was a true adaptation, which depended no longer on direct visual cues, such as disparity. Furthermore, Lemij and Collewijn (1991b) showed that nonconjugate adaptations could be fairly complete in as little as $1 \mathrm{hr}$ of spectacle-wearing. They also demonstrated that post-saccadic drift of horizontal saccades was appropriately adapted to the requirements of the anisometropic spectacles. Smooth-pursuit eye movements showed similar nonconjugacies as saccades after adaptation to anisometropic spectacles (Lemij \& Collewijn, 1991a, b).

Interestingly, horizontal and vertical saccades did not invariably adapt to the same extent: adaptations along either of these meridians could be more complete than those along the orthogonal one. This finding suggests that adaptations along one meridian may take place independently from those along another one. Such 
meridian-specificity would greatly add to the degree of freedom of the oculomotor system in adjusting adequately to a non-uniform pressure for adaptation along the various meridians. Such pressure might result from local mechanical changes within the orbit caused by ageing, disease, fatigue, of from sclective neural damage. Appropriate, meridian-specific adaptation would contribute to proper oculomotor control throughout a lifetime. Meridian-specific adaptation of saccades was demonstrated in man and in monkey by Deubel (1987). He had humans and monkeys follow a target that jumped in a specific direction. During the saccade, the target jumped again, consistently to another position. After sufficient training, the humans and monkeys made their initial saccade directly towards the second target position. This adaptation transferred partially to adjacent meridians, becoming smaller as the difference in orientation of the meridians grew larger. The experiments by Deubel (1987) did not address the question of whether such meridian-specific adaptation could be induced specifically in one eye only.

In the present experiments we shall demonstrate that the wearing of anisometropic spectacles may bring about nonconjugate, meridian-specific adaptations of saccades, and also of smooth-pursuit eye movements. These results have been published before as an abstract (Lemij \& Collewijn, 1989b) and as part of a doctoral thesis (Lemij, 1990).

\section{METHODS}

Two sets of experiments were carried out on different groups of subjects. Both dealt with nonconjugate, meridian-specific adaptation of saccades to the shortterm wearing of anisometropic spectacles. Specifically, we examined whether such adaptations could take place along one meridian, without affecting the conjugacy of saccades made along the orthogonal meridian. We determined in passing whether any such adaptations were also expressed in smooth-pursuit eye movements. The first set of experiments addressed the horizontal and vertical meridians, whereas the second set focused on oblique meridians.

\section{Subjects}

In the first and second set of experiments, 9 and 10 subjects took part, respectively. None of these had any history of ocular or oculomotor pathology. Several subjects had refractive anomalies, which were corrected by rigid contact lenses. Visual acuities of all subjects were, with their own contact lenses if they had any, 5/5 or better in either eye. Because we assumed that good binocular vision was mandatory for adequate, nonconjugate adaptation, we tested all our subjects on stereopsis. They all passed this test (TNO test for stereoscopic vision; thresholds $120 \mathrm{sec}$ arc or better).

\section{Adaptation}

All subjects. were supplied with anisometropic spectacles that contained a planocylindrical spectacle- lens $(-3.0 \mathrm{D})$ in front of the right eye. Along the meridian parallel to the axis of such a cylindrical lens, the size of the visual image remains virtually unaffected, whereas along the orthogonal meridian the visual image is reduced in size, due to the prismatic effects of the negative cylinder. This reduction in size was of the order of $6 \%$. The left eye was not supplied with a lens.

The subjects wore these anisometropic spectacles for $7 \mathrm{hr}$ continuously. During this adaptation-period, they carried out their normal daily activities. For the first set of experiments, the cylinder-axis was positioned horizontally on one occasion, thus requiring nonconjugate adaptations along the vertical meridian. On another day, the cylinder-axis was positioned vertically. Obviously, this condition called for horizontal nonconjugate adaptations, without any such pressure along the vertical meridian. For the second set of experiments, the cylinder-axis was positioned at $45 \mathrm{deg}$, according to customary notation. Therefore, the visual image was reduced in size from the bottom-left to the top-right, as experienced by the subject. Arbitrarily, we called this meridian the $45 \mathrm{deg}$ meridian. Along the orthogonal, or 135 deg meridian, the spectacles did not provide any pressure for adaptation. In both sets of experiments, we ran the actual measurements immediately following the adaptation-period. On separate days, baseline recordings were made without any adaptation. The order of all these sessions was randomized, in every set of experiments, for every subject. To avoid carry-over of adaptation effects, successive sessions were separated by at least 3 days of normal, every-day conditions.

\section{Experimental procedures}

During the actual measurements, the subjects wore the anisometropic spectacles. The experimental design was similar to the one described before (Lemij \& Collewijn, $1991 \mathrm{a}, \mathrm{b})$. In short, subjects made repetitive saccades between stationary targets that were projected symmetrically around the straight-ahead position onto a white screen. This screen was moulded as a (toroid) isovergence surface, such that under normal viewing all points on the screen subtended an equal vergence angle of about 4.7 deg (see Lemij \& Collewijn, 1991a). In this way, changes in target vergence were exclusively caused by the anisometropic spectacles, and not by any displacement of the targets on the screen. We employed stationary targets, because they yield more accurate saccades than a jumping target (Lemij \& Collewijn, 1989a). The targets consisted of bright-red $\mathrm{He}-\mathrm{Ne}$ laser spots, with a dia of approx. $5 \mathrm{~min}$ arc. In the first set of experiments, the target separations were $5,10,20$ or $30 \mathrm{deg}$, both along the horizontal and along the vertical meridian. In the second set of experiments, dealing with nonconjugate adaptations along oblique meridians, the targets were presented along both the $45 \mathrm{deg}$ and the $135 \mathrm{deg}$ meridian, with target separations of $10,14,30$ and $42 \mathrm{deg}$. The saccades were made to a sound beating steadily at a comfortable pace of 45 per min. Data collection lasted $12 \mathrm{sec}$ for every trial, and was initiated by the subjects themselves by pressing a button. The 
order of the trials was randomized for every subject. Viewing was binocular or monocular with either eye. Subjects were requested to refrain from blinking, in order to prevent the occurrence of associated eye movements (Collewijn, Van der Steen \& Steinman, 1985). Following the saccade-experiments, the subjects tracked a single target that moved across the screen in a circle at a low, constant tangential velocity (about $11 \mathrm{deg} / \mathrm{sec}$ ). Again, viewing was binocular or monocular with either eye. During all measurements, the head was stabilized by means of forehead- and chin-support. At the end of all trials, we had the subjects monocularly fix targets with known positions by the right eye, viewing through the spectacles, to measure the target separations as viewed by that eye. Monocular fixations made by each of the two eyes without spectacles were also recorded for calibration purposes.

\section{Data-acquisition and data-analysis}

Eye movements were recorded binocularly by means of magnetic search coils (Collewijn, Van der Mark \& Jansen, 1975) in a Robinson type magnetic field configuration (Robinson, 1963). The recordings were sampled with 12-bit precision and stored on disk. The overall bandwidth was estimated at $125 \mathrm{~Hz}$. Our recording technique allowed only for the recording of the horizontal and vertical components of all eye movements. Therefore, the trajectories of the oblique saccades made in the second set of experiments were later digitally reconstructed from corresponding sample-points of the individual horizontal and vertical recordings. These reconstructed, oblique saccades were then, again, broken down into two orthogonal components: this time, however, not along the horizontal and vertical meridians, but along their oblique, 45 and 135 deg meridians. This coordinate-transformation allowed for the main components of the oblique saccades to be studied similarly as horizontal and vertical saccades along cardinal meridians. When the spectacles were taken off to place the search coils onto the eyes, care was taken that one eye remained covered at all times, to prevent any symmetrical visual input, that might undo the effects of adaptation.

As described before (Lemij \& Collewijn, 1991a,b), small re-calibrations were digitally carried out off-line to speed up the recording sessions. In addition, the coordinates of all eye-positions were digitally transformed into Helmholtz's coordinates, because the latter best serve a clear comparison in position between the two eyes (see Carpenter, 1988, p. 150). For practical reasons, Helmholtz's coordinates could not be obtained directly. Saccades were analyzed off-line according to standard criteria by a computer-program, which calculated the size and the direction of each primary saccade, as well as the mean post-saccadic drift velocity. These standard criteria have been presented before (Lemij \& Collewijn, 1991a); briefly, saccadic onset and offset were determined by velocity thresholds of $15 \mathrm{deg} / \mathrm{sec}$, and post-saccadic drift was measured over a period of $68 \mathrm{msec}$, which began $25 \mathrm{msec}$ after saccadic offset.
The same standard criteria were adopted for the oblique saccades. Slight displacements of the spectacles on the head were likely to occur during the running of the experiments. Such displacements would affect the absolute positions of the targets as seen by the right eye. We therefore refrained from determining absolute fixation accuracy, and limited our analysis to changes in the size of the saccades. A statistical software package (SPSS-X) was used to carry out a multivariate analysis of variance (MANOVA). To obtain normal distributions and homogeneous variances, as required by this package, saccadic magnitudes, as well as post-saccadic drift velocities were transformed logarithmically.

An analysis of possible measurement-errors was carried out. It turned out that, due to a systematic distortion in the recording equipment, eye-positions of the right eye along the $45 \mathrm{deg}$ meridian were underestimated by about $1.5 \%$, whereas eye-positions of the same eye along the orthogonal, $135 \mathrm{deg}$ meridian, were overestimated by about $1 \%$. Similar deviations for the left eye were $<0.5 \%$, along either of the oblique meridians. Therefore, the absolute differences in saccadic size between the two eyes per se were not a trustworthy measure of the degree of nonconjugate adaptation. For that reason, we will only compare saccadic sizes before adaptation (baseline) with those after adaptation. Such differences will be very little affected by this systematic measurement-error.

\section{RESULTS}

\section{Nonconjugate adaptation of horizontal or vertical saccades}

Nonconjugate saccades had developed in all subjects during the $7 \mathrm{hr}$ of conditioning to the anisometropic spectacles in the first set of experiments, which aimed at nonconjugate, meridian-specific adaptation along the cardinal meridians. The nonconjugacies were highly meridian-specific $\left(F_{2,16}=314.3 ; P<0.0001\right)$, in accordance with the requirements of the spectacles. Typical recordings of one subject, which illustrate these specific adaptations for either meridian, are shown in Fig. 1. The left panel displays binocular recordings of horizontal and vertical saccades made before adaptation. The middle panel shows asymmetrical adaptation along the horizontal meridian, induced by a horizontally minifying lens. Horizontal saccades of the right, adapted eye, were in this case about $1.9 \mathrm{deg}$ smaller than those of the left eye (nominal target separation $30 \mathrm{deg}$ ).

The conjugacy of the vertical saccades, however, was virtually unchanged. When the same subject wore, on another day, spectacles with the same lens in front of the right eye, with the lens-axis rotated by $90 \mathrm{deg}$, so that minification was maximal along the vertical meridian, vertical saccades become nonconjugately adapted to about the same degree as the horizontal adaptation had been, while horizontal saccades remained conjugate. The recordings of Fig. 1 were made during binocular viewing. With one eye covered, the same, highly meridian-specific nonconjugate adaptations were present, although the 
Base line
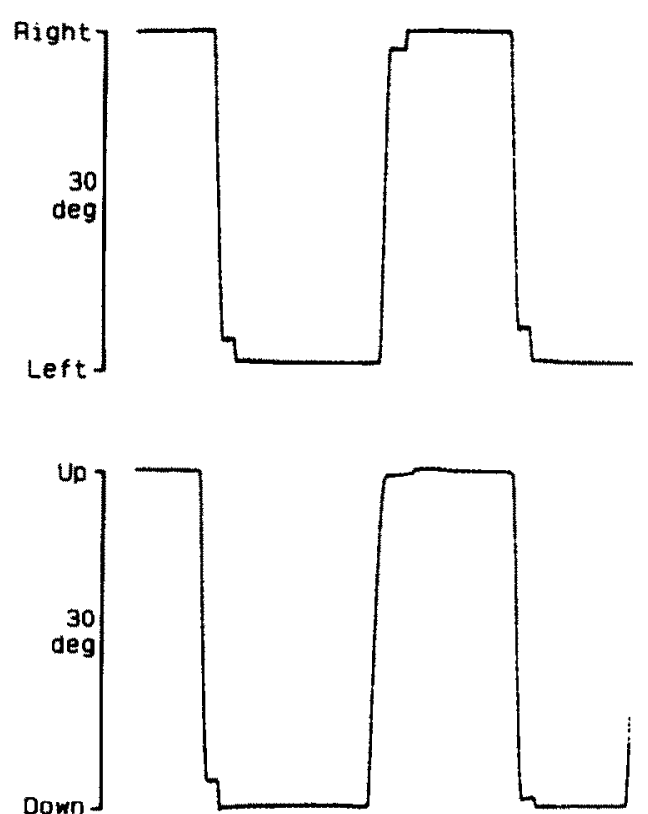

Horizontally adapted

Horizontal saccades

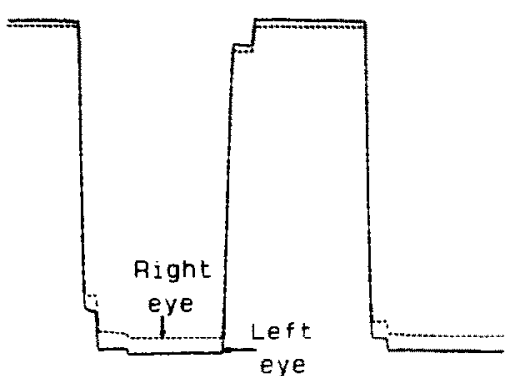

Vertical saccades

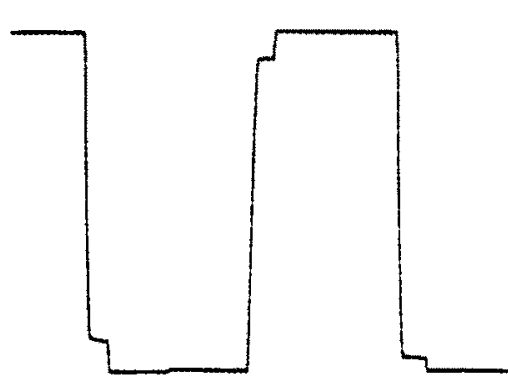

FIGURE 1. Typical recordings of horizontal and vertical saccades of one subject, made during binocular viewing. Left panel, baseline recordings; middle panel, horizontal nonconjugate adaptation; right panel, vertical nonconjugate adaptation.

differences in saccadic size between the two eyes were somewhat smaller.

To quantify the magnitude of these nonconjugate adaptations, and also to indicate their meridianspecificity, we present mean differences in saccade size between the two eyes for horizontal and for vertical saccades in Fig. 2 (for binocular viewing) and Fig. 3 (for monocular viewing). For comparison, baseline values have been added. The difference in saccadic size between the two eyes was calculated as the saccadic magnitude of the left eye minus the magnitude of a concomitant saccade of the fellow, right eye. Baseline recordings confirmed the essential conjugacy of saccades under normal conditions, with yoking being better for vertical saccades than for horizontal saccades, and better during binocular viewing than during monocular viewing.
Figures 2 and 3 show clearly that adaptive nonconjugacies occurred over the full range of target separations (5 $30 \mathrm{deg}$ ) in the appropriate meridian only; the degree of yoking in the orthogonal (non-adapted) meridian remained, after adaptation, essentially the same as in the baseline condition. The magnitude of the adaptive size-difference, or vergence, was, for the saccades made during binocular viewing, of the order of $4-7 \%$ of the target separation. This amounted to up to about 2 deg for the largest target separation, which was nominally $30 \mathrm{deg}$. (The term "vergence" is used here in a technical sense, meaning simply the change in angle between the lines of sight, without implications with regard to a specific role of the vergence system in these adaptations.) For the smallest target separation ( $5 \mathrm{deg}$ ), changes in the degree of yoking were as small as about
Horizontal saccades

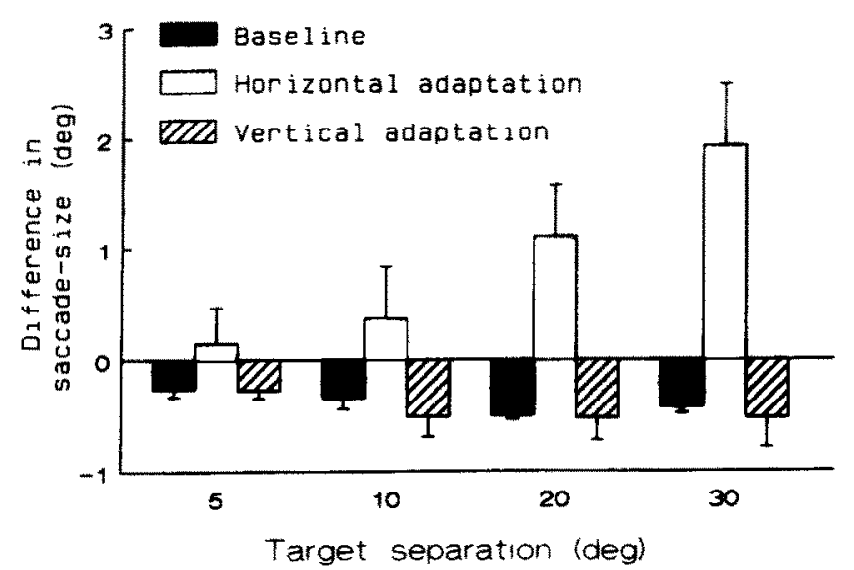

Vertical saccades

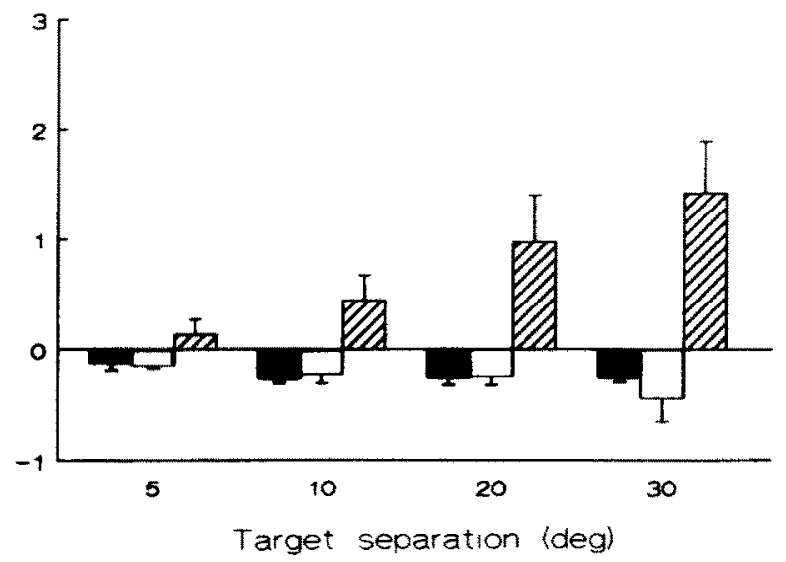

FIGURE 2. Mean differences in saccade size between the two eyes, expressed in deg ( $\mathrm{SD}$ ), for horizontal saccades (left panel) and vertical saccades (right panel). Binocular viewing. For comparison, baseline values have been added. 
Horizontal saccades

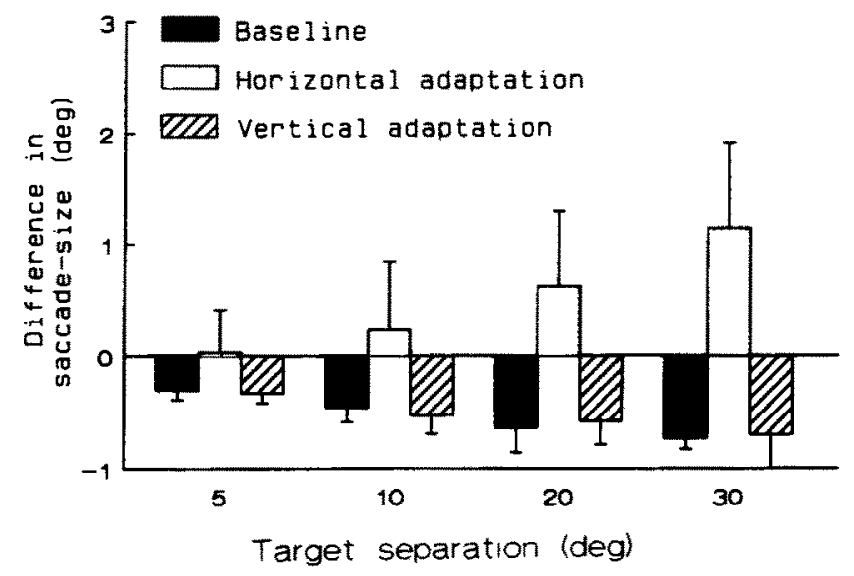

Vertical saccades

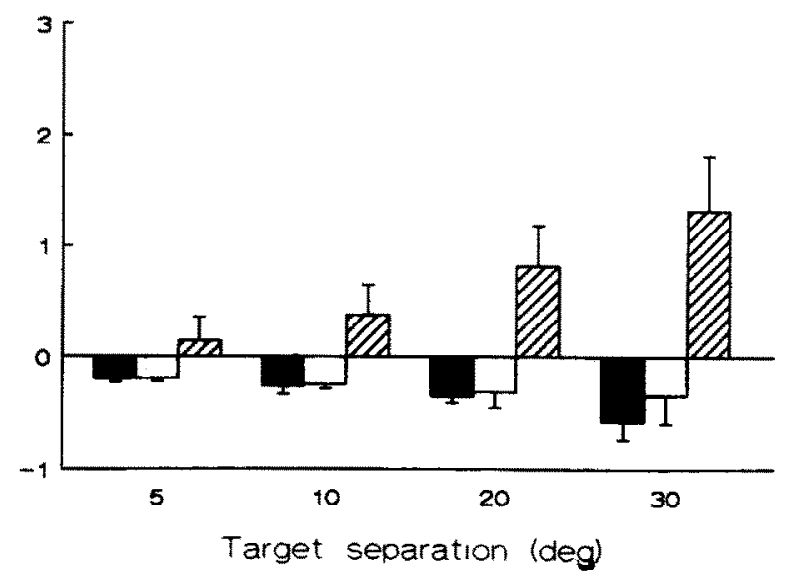

FIGURE 3. Same as Fig. 2, for monocular viewing with either eye.

$15 \mathrm{~min}$ arc. The adaptive nonconjugacies were, on average, about as large for horizontal saccades as for vertical saccades. However, somc subjects were better adapted along one meridian (either horizontal or vertical) than along the orthogonal one. Such preferences varied also unsystematically with the target separation. When one eye was covered, the nonconjugacies were, on average, $30 \%$ smaller but they remained equally meridian-specific as during binocular viewing (compare Fig. 2 with Fig. 3). This indicates that the meridian-specificity was relatively permanent, and not the immediate result of visual information obtained from both eyes shortly before saccadic onset. The degree of nonconjugacy was, on average, similar for monocular viewing with either the left or the right eye. Therefore, we pooled the data of the two monocular viewing conditions.

To assess the degree of nonconjugate adaptation for either cardinal meridian, and for either viewing condition, we measured the vergence-deficits, calculated as the required change in vergence of concomitant saccades minus their actual change in vergence. These results are summarized in Fig. 4. The nonconjugate adaptations along the horizontal and vertical meridians were almost complete during binocular viewing: vergence-deficits (Fig. 4) averaged only about $0.3 \mathrm{deg}$ for all four target separations along these two meridians, with maximal values of about $0.6 \mathrm{deg}$ for $30 \mathrm{deg}$ vertical saccades.
None of our subjects in the first set of experiments perceived any double-images after $7 \mathrm{hr}$ of adaptation, which indicates that all vergence-deficits remained within the fusional limits. With both eyes viewing, the overall degree of nonconjugate adaptation was about $73 \%$ of what was called for by the spectacles. When one eye was covered, the overall degree of asymmetrical adaptation dropped to about $52 \%$, with vergence-deficits reaching maximal values of about $1 \mathrm{deg}$. These values are similar to those found in habitual wearers of anisometropic spectacles (Lemij \& Collewijn, 1991a). Figure 4 suggests that on average, during monocular viewing, the degree of asymmetrical adaptation equalled a fixed percentage of what was called for by the spectacles, because the vergence-deficits increased approximately linearly with larger target separations. This trend in the pooled data was much less clear in the vergence-deficits of individual subjects, which showed more variability.

\section{Nonconjugate adaptation of oblique saccades}

Nonconjugate adaptations of oblique saccades occurred in all subjects participating in the second set of experiments. These nonconjugate adaptations were specific for the appropriate, $45 \mathrm{deg}$ meridian $\left(F_{1,9}=66.9\right.$; $P<0.0005)$. Along the orthogonal, $135 \mathrm{deg}$ meridian, no nonconjugate changes whatsoever developed during $7 \mathrm{hr}$ of adaptation. This result satisfied the requirements
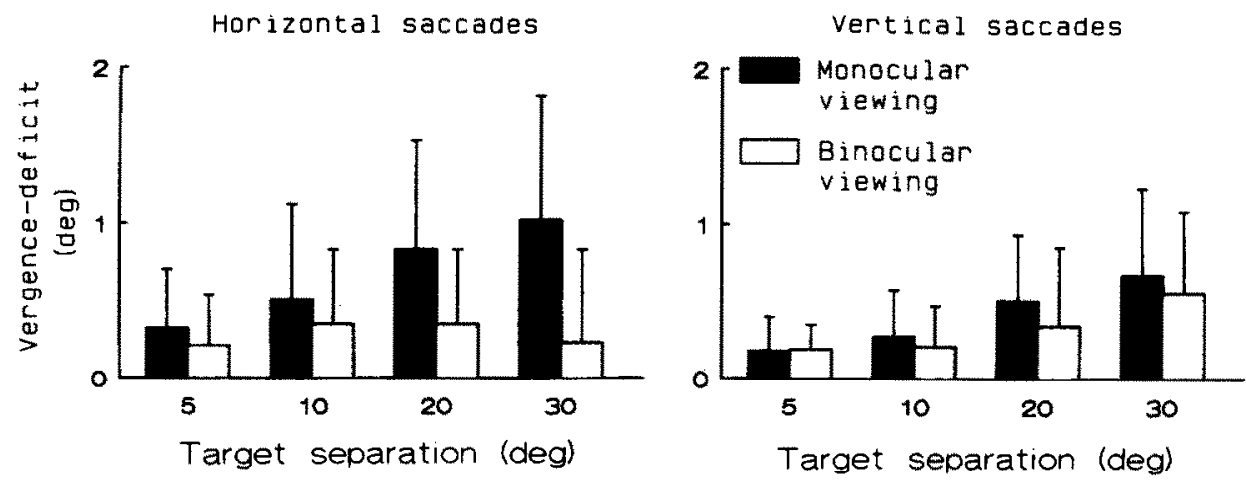

FIGURE 4. Mean vergence-deficits (+SD) for horizontal saccades (horizontally adapted) and for vertical saccades (vertically adapted) during binocular and monocular viewing. 


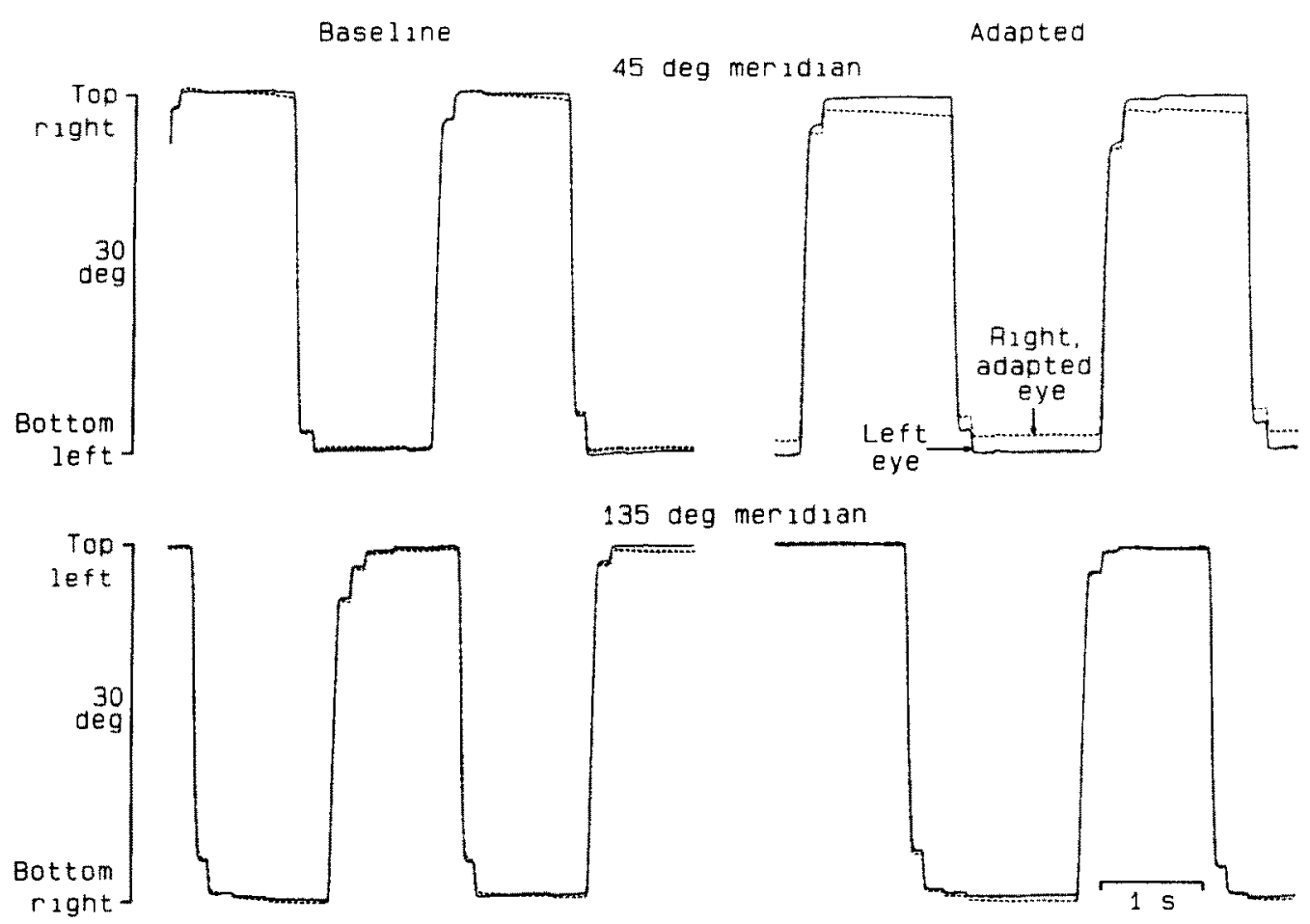

FIGURE 5. Typical examples of binocular oblique saccades made along the $45 \mathrm{deg}$ meridian (upper panels) and along the $135 \mathrm{deg}$ meridian (lower panels), after coordinate transformation of the horizontal and vertical recordings to oblique coordinates. Left panels, baseline recordings; right panels, after adaptation of the right eye to minification in the 45 deg meridian. Viewing was with the left eye.

of the spectacles. Figure 5 displays some typical oblique saccades (shown after transformation of the recordings to an oblique coordinate system) both before and after adaptation. This figure shows that, before adaptation (left panels), saccades were conjugate along either oblique meridian ( 45 or $135 \mathrm{deg}$ ). After adaptation to the oblique cylinder, however, the oblique saccades, made along the 45 deg meridian, were smaller by about $2.1 \mathrm{deg}$ in the right eye than in the left eye (target separation, nominally $30 \mathrm{deg}$ ). At the same time saccades made along the orthogonal, 135 deg meridian had remained conjugate. Note that the recordings of Fig. 5 were made during monocular viewing with the left eye. Thus, they illustrate that these nonconjugate adaptations were retained in the absence of direct disparity information.

To quantify these nonconjugate adaptations, we measured again the difference in size between concomitant saccades of the two eyes, calculated as the magnitude of saccades made by the left eye minus the magnitude of saccades of the right (adapted) eye. Because variations in the degree of nonconjugate adaptation between subjects were very small, we shall focus on the main trends. Figure 6 presents mean values for the differences in saccade-size between the two cyes, both before and after adaptation, for the four various target separations. Distinctions have also been made between the various viewing conditions (binocular viewing vs monocular viewing with either eye). As the sizedifferences did not significantly depend on whether the right eye or the left eye was viewing, the data of these two monocular viewing conditions were pooled. As expected, conjugacy along either of the oblique meridians was good in the baseline condition. After adaptation along the $45 \mathrm{deg}$ meridian, marked sizedifferences developed along this meridian which became larger at larger target separations $\left(F_{3.27}=46.8\right.$; $P<0.0005$; Fig. 6, left panels). During binocular viewing, the right, adapted eye made saccades along the $45 \mathrm{deg}$ meridian, that were about $6 \%$ smaller than before adaptation (all four target separations), whereas the saccades of the left eye had not changed in size. The magnitudes of these adaptive nonconjugacies could, for the binocular viewing condition, become as large as $2.6 \mathrm{deg}$, compared to baseline-values (target separation, nominally $42 \mathrm{deg}$ ). Along the orthogonal, $135 \mathrm{deg}$ meridian, differences in saccade-size between the two eyes remained as small as before adaptation (Fig. 6, right panels). Importantly, Fig. 6 also demonstrates that, when viewing was monocular, this meridian-specificity was still present, although in this condition the magnitudes of the adaptive nonconjugacies were about $30 \%$ smaller than during binocular viewing.

As a next step, we determined how complete the oblique nonconjugate adaptations were, by examining the vergence-deficits present at the end of each primary saccade, for every viewing condition. Vergence-deficit was defined and calculated as before. Mean values of vergence-deficits are presented in Fig. 7. The data are confined to the $45 \mathrm{deg}$ meridian, along which the nonconjugate adaptation was required. It is clear from this figure that the nonconjugate adaptations were almost complete during binocular viewing, vergence-deficits being as small as about $0.3 \mathrm{deg}$ across the full range of target separations. This corresponded well with the fact that most subjects experienced fusion of the 
45 deg meridian

135 deg meridian
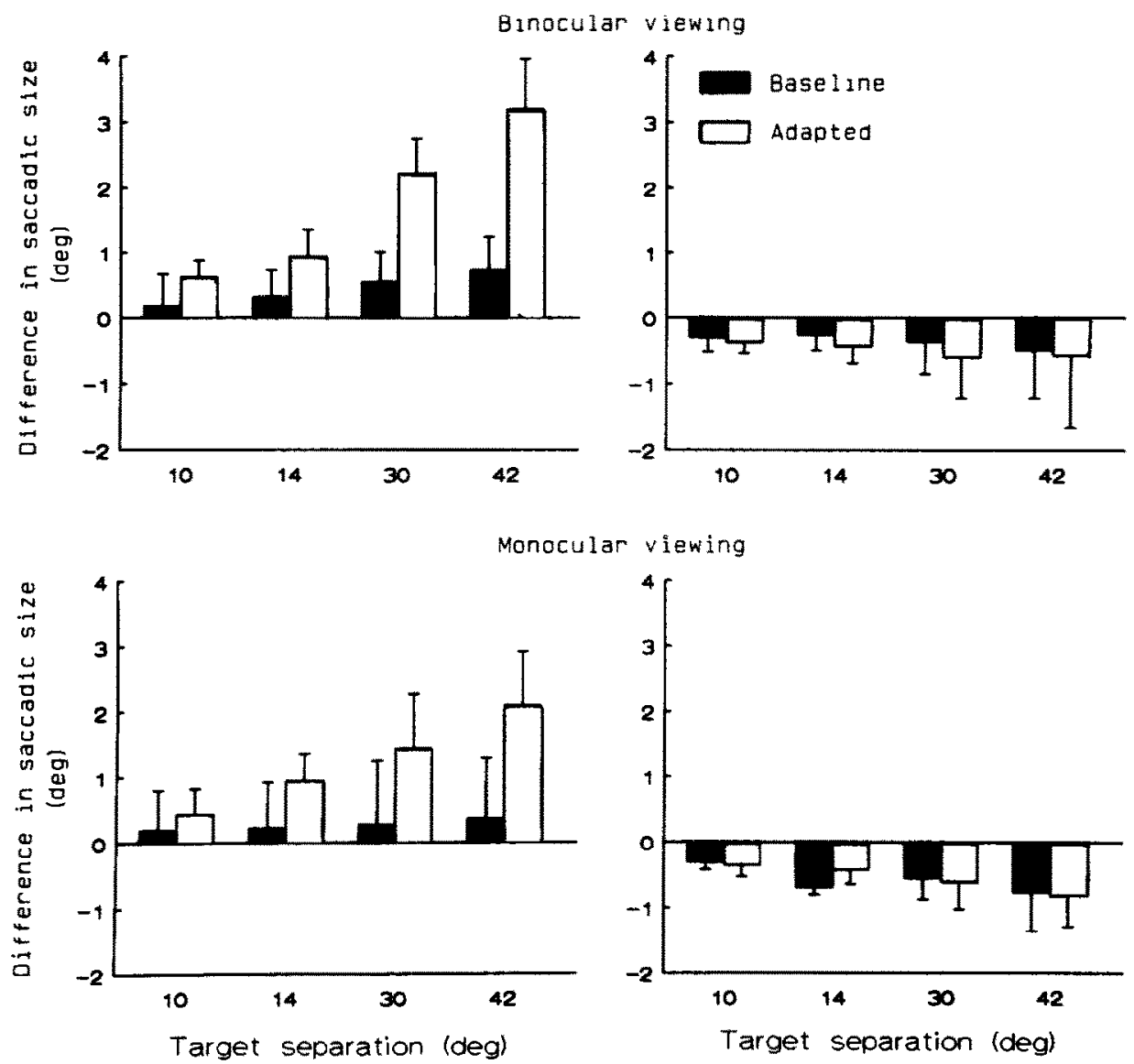

FIGURE 6. Mean differences in saccadic size between the two eyes $(+\mathrm{SD})$ after adaptation of the right eye in the 45 deg meridian, computed as the magnitude of saccades of the left eye minus the magnitude of concomitant saccades of the right eye, for either meridian (left panel, $45 \mathrm{deg}$ meridian; right panel, $135 \mathrm{deg}$ meridian) at the four various target separations. Baseline-data have been included. Top panels relate to the binocular viewing conditon. Bottom panels represent data obtained during monocular viewing with either eye.

targets. Although other subjects clearly saw two distinct images of each target, one being normal and the other distorted through the action of the cylindrical lens, these images were always seen superimposed, in agreement with the small vergence-deficits. When one eye was covered, the nonconjugate adaptations were less complete $\left(F_{2,18}=14.6 ; P<0.0005\right)$, with vergence-deficits becoming 2- to 4-fold larger than during binocular viewing, and reaching about $1.5 \mathrm{deg}$ for the largest target separation of, nominally, $42 \mathrm{deg}$. The nonconjugate

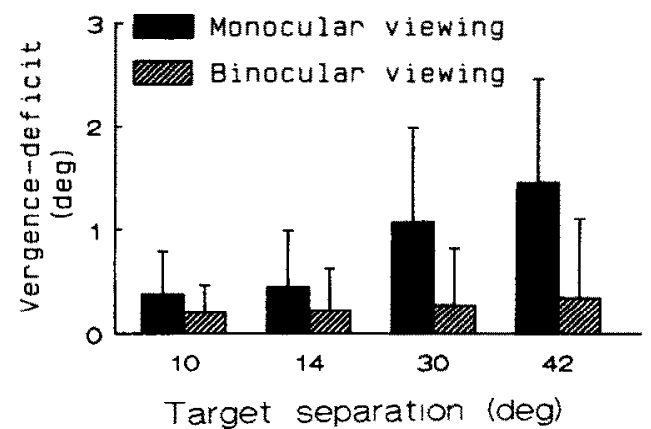

FIGURE 7. Mean vergence-deficits (+SD) for saccades made along the adapted, $45 \mathrm{deg}$ meridian at the four various target separations. Data relate to monocular viewing with either eye (solid bars) and to binocular viewing (hatched bars). adaptations along the $45 \mathrm{deg}$ meridian were equally complete as those of horizontal and vertical saccades.

\section{Post-saccadic drift}

Asymmetrical adaptation of post-saccadic drift was only observed for horizontal saccades and occurred in all subjects participating in the set of experiments on adaptation along the horizontal meridians $\left(F_{2,16}=25.5\right.$; $P<0.0005$ ). We prefer using the term "asymmetrical adaptation" in the present context, rather than nonconjugate adaptation, because it is well-known that normal postsaccadic drift is not entirely conjugate (see e.g. Kapoula, Robinson \& Hain, 1986). The term asymmetrical signifies that the adaptations were unequal for the two eyes. The plastic, asymmetrical changes in post-saccadic drift that we found were such that they reduced the residual vergence-deficits at saccadic offset. This was true for binocular viewing, and, to the same extent, also for monocular viewing with either eye. We shall, therefore, not distinguish between these viewing conditions. As mentioned before, the mean postsacadic drift velocity was determined in the interval of 25-93 msec after saccadic offset. For adducting saccades (Fig. 8, upper panel), the predominantly onward drift of the left, normal eye increased in mean velocity by about 

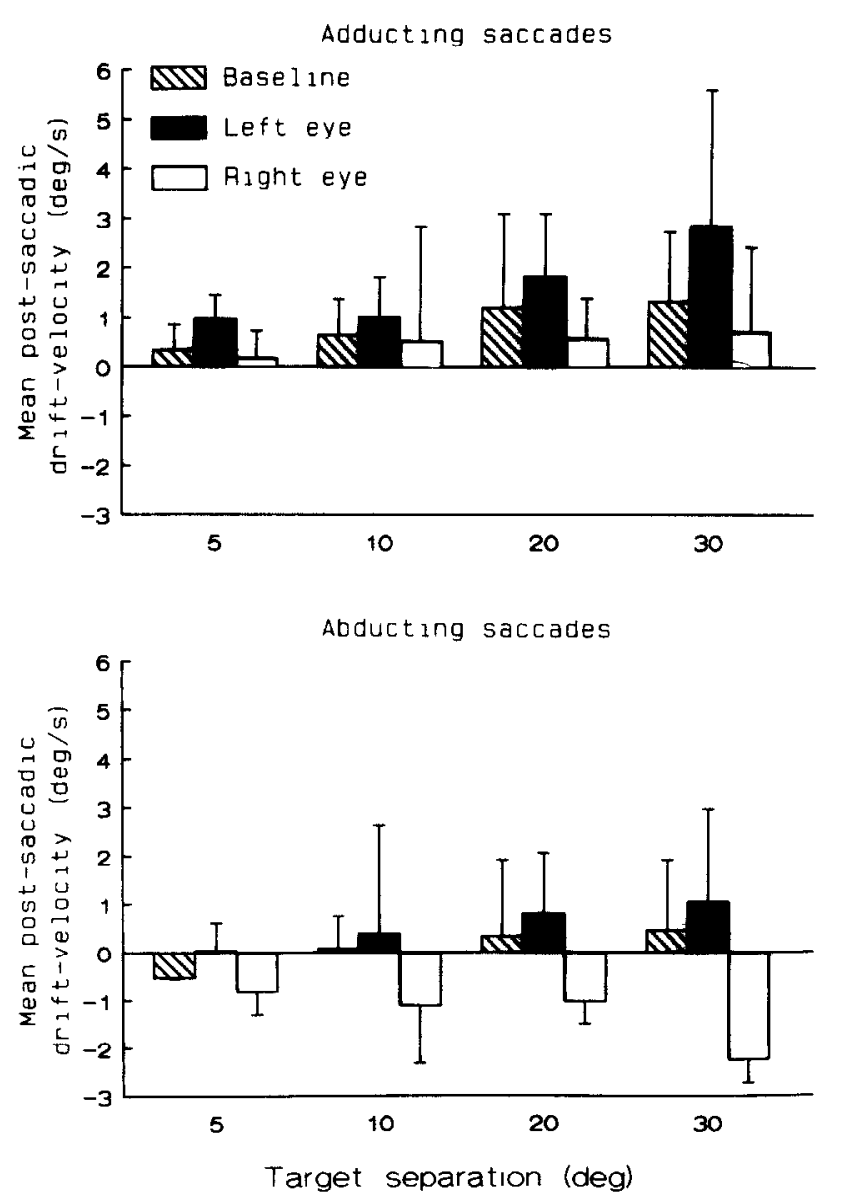

FIGURE 8. Mean post-saccadic drift velocities (+SD) for adducting saccades (upper panel) and abducting saccades (lower panel). The data relate to binocular viewing, both before and after nonconjugate adaptation along the horizontal meridian (minifying lens over right eye: plano $=\mathrm{C}-3.0 \times 90 \mathrm{deg}$ ). Positively signed post-saccadic drift indicates onward drift, whereas negatively signed drift denotes backward drift.

\section{Smooth-pursuit}

Similarly to the saccades, smooth-pursuit eye movements, made to the circularly moving target, were adapted nonconjugately in all subjects. As with saccades, these adaptations occurred essentially along the appropriate meridian only, under both binocular and monocular viewing conditions. Figure 9 presents some typical recordings, made during binocular viewing, which illustrate these plastic changes along the horizontal and vertical meridians, as well as along thc oblique, $45 \mathrm{deg}$ meridian. Slight deviations from the appropriate meridian did occur, however, in most subjects. During monocular viewing with the eye not provided with a cylindrical spectacle-lens, these deviations were usually larger than during binocular viewing. Maximum values of such deviations were about $15 \mathrm{deg}$. We quantified the degree of nonconjugate, meridian-specific adaptation, by measuring the smallest diameter of the circular movements of either eye within a range of $15 \mathrm{deg}$ to either side of the appropriate meridian, i.e. the meridian which supplied the maximum adaptive stimulus. We also measured the diameter of the circular movement of either eye along the meridian orthogonal to the previous one. From these data we calculated the nonconjugacies along the specified meridians. As a next step, we determined the nonconjugacies that were actually required by the spectacles from the recordings made of the viewing eye during monocular viewing. By subtracting the actual nonconjugacies from the required nonconjugacies along the meridians under consideration, we computed the vergence-deficits as we did for the saccades. The nonconjugate adaptations along the $45 \mathrm{deg}$ meridian were just as complete as those along either cardinal meridian, during both binocular and monocular viewing
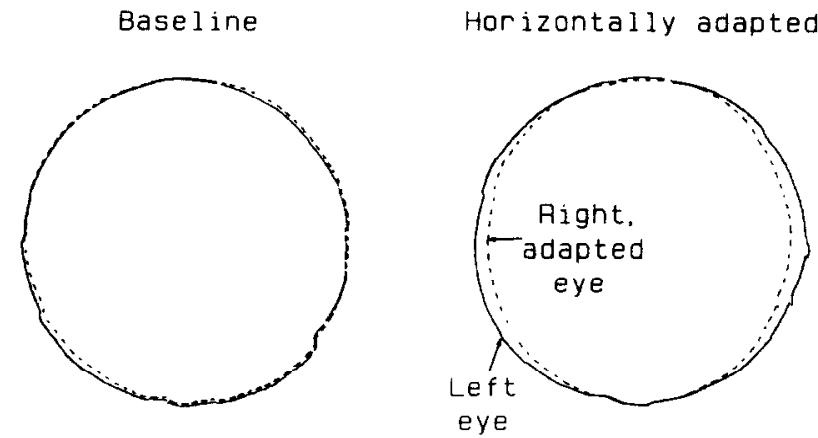

$0.6 \mathrm{deg} / \mathrm{sec}$. At the largest target separation (nominally $30 \mathrm{deg}$ ) the drift velocity had increased 2-fold, up to about $3 \mathrm{deg} / \mathrm{sec}$. The right eye also drifted predominantly in the onward direction after adducting saccades, but its drift velocity had dropped by about $0.3 \mathrm{deg} / \mathrm{sec}$, compared to baseline values. For abducting saccades, the asymmetrical adaptation of post-saccadic drift was characterized by a change in direction of the right eye: instead of the normal, onward drift, the right eye now drifted backward. Its mean drift velocities were on the order of $0.5 \mathrm{deg} / \mathrm{sec}$ over the full range of target separations. When the fellow eye made an abducting saccade, this eye drifted with a velocity about twice as high as before asymmetrical adaptation. All these results suggest that the pulse-step ratio, which characterizes the motor commands to the external eye muscles, was adaptively changed for horizontal saccades, when the spectacles called for asymmetrical, horizontal adaptation. When vertical adaptation was required, no changes in the yoking of post-saccadic drift occurred. In addition, we found no asymmetrical adaptation whatsoever in the post-saccadic drift of oblique saccades.
Vertically adapted

Obliquely adapted
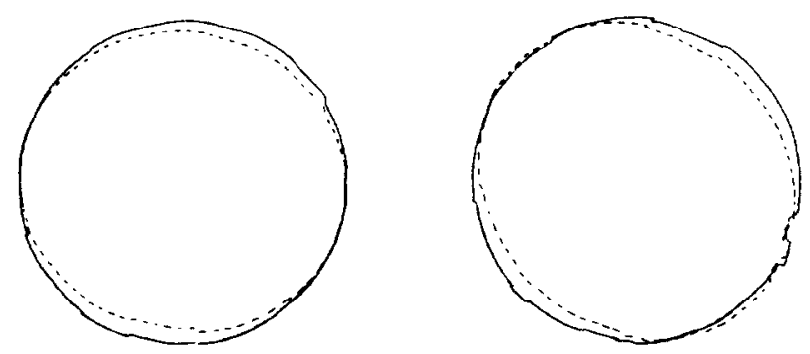

FIGURE 9. Typical binocular recordings made during the smoothpursuit task, for the three various, nonconjugately adapted meridians. Baseline recordings have been included. Viewing was binocular. 
with the eye. Vergence-deficits were on the order of $0.2 \mathrm{deg}$ during binocular viewing and about $1.2 \mathrm{deg}$ during monocular viewing with either eye. In the experiment on adaptive nonconjugacies along the cardinal meridians, some subjects displayed a somewhat more complete adaptation along one meridian (either horizontal or vertical) than along the orthogonal one. These data compare remarkably well with those we found in habitual wearers of anisometropic spectacles (Lemij \& Collewijn, 1991a).

\section{Perception}

After the first few hours of wearing the anisometropic spectacles, the subjects perceived the visual world as distorted. With the cylinder-axis positioned vertically, thus reducing the visual world along the horizontal meridian, objects on the left side of the visual field appeared larger and farther away than those on the right. Many subjects reported that they misjudged distances, and, as a consequence, occasionally bumped accidentally into objects on their left side. Also, horizontal surfaces appeared to be tilted: ceilings seemed to go down towards the right side, whereas floors gradually looked higher on that side. With the lens-axis positioned horizontally, the distortions were exactly opposite: objects on the right side of the visual field now appeared larger and farther away than on the left, and horizontal surfaces were tilted in the opposite direction. With the cylinder-axis tilted obliquely, the distortions consisted of a shrinking of objects in the upper visual field, as well as an increase in distance between those objects and the subject. These distortions, which were observed also to some degree with anisometropias brought about by spherical lenses (Lemij \& Collewijn, 1991b), confirm the well-known descriptions by Ogle (1962), who coined the terms "induced effect" (for vertical magnifications) and "geometrical effects" (for horizontal magnifications), observed as distortions of a "leaf room". The illusions did not lessen within $7 \mathrm{hr}$ of conditioning, but disappeared completely in about $2 \mathrm{~min}$ after the spectacles were taken off. We did not endeavour to quantify these phenomena, because that would be beyond the scope of our present oculomotor research, but mention them nonetheless, since they were very striking.

\section{DISCUSSION}

\section{Meridian-specificity of saccadic adaptation}

The principal finding in these experiments is that nonconjugate adaptations of saccades and smoothpursuit eye movements can be specific for either the horizontal or the vertical meridian, or for an oblique meridian. These results suggest that nonconjugate adaptation can be specific for any meridian. Since the nonconjugacies were also present during monocular viewing, the meridian-specific plastic changes proved to be incorporated in the programme controlling binocular saccades, so that it had become independent of immediate visual input. These results demonstrate yet another VR $32 / 3-C$ aspect of the capability of oculomotor control to adjust to specific changes in the visuo-motor relations. The speed of these adaptations was remarkable: they were equally complete as in habitual wearers of anisometropic spectacles after as little as $7 \mathrm{hr}$ of adaptation (for a comparison, see Lemij \& Collewijn, 1991a). The persistent distortions of the outside world which our subjects perceived, suggest that these motor adaptations take place more readily than a re-mapping of sensory perception. The speed and specificity of the nonconjugate adaptations we demonstrated suggest that the oculomotor system is capable of coping adequately with the various changes at various locations that are likely to occur throughout a lifetime. Only by adapting specifically to such, generally non-uniform, changes can eye movements remain accurate.

Obviously, these adaptations occurred because there was a pressure for such specific changes. Other types of highly specific saccadic adaptations have been demonstrated in the past. For horizontal saccades, a pressure for (conjugate) adaptation in only one direction (either to the left or to the right) induces appropriate, directionspecific adaptive changes. This was shown in patients with unilateral palsies of a medial or lateral rectus muscle (Kommerell, Olivier \& Theopold, 1976; Abel, Schmidt, Dell'Osso \& Daroff, 1978; Optican, Zee \& Chu, 1985), but also for saccades made in one direction towards a target that jumped consistently during the execution of that saccade, to another position, in order to induce direction-specific changes in saccadic size (Miller, Anstis \& Templeton, 1981; Duebel, Wolf \& Hauske, 1986). In all these experiments, the directionspecific adaptations did not transfer to the opposite direction (possible transfer to other meridians was not tested). This brings up the question how meridianspecific the adaptive properties of the saccadic sybsystem, and of other oculomotor systems alike, can be. In case of the muscle-pareses, direction-specific adaptations presumably also developed in closely adjacent meridians. It would be difficult to distinguish, however, whether such a broadening of adaptive changes to adjacent meridians resulted from poor meridianspecificity, or from a specific need for such broader adaptation, because saccades along those adjacent meridians would also involve the action of the weakened eye muscle. Therefore, adaptive changes would be called for in proportion to that action.

In our present experimental design, the whole visual world of one eye was reduced in magnitude along one dimension. Therefore, in case of a size-reduction which was maximal along the horizontal meridian, there was not only a pressure for purely horizontal saccades to adapt nonconjugately, but also for the horizontal components of all oblique saccades. For a cylindric lens, power and magnification factor vary as a function of the meridional orientation as follows:

$$
P_{\phi}=P_{\mathrm{cyl}} \cos ^{2} \phi
$$

in which $P_{\text {cyl }}$ is the power of the cylinder in the axis of maximum curvature, and $P_{\phi}$ is the power in a meridian 
oriented at an angle $\phi$ to the axis of maximum curvature (this relation follows from Euler's general formula; see Southall, 1961, p. 146). Thus, the meridional distribution of magnification was relatively broad in our experiments, and even if we had recorded saccades in many intermediate directions, our present experimental design would not have been appropriate to assess how narrowly tuned meridian-specific nonconjugate adaptation may be. The relatively broad meridional distribution of our adaptations is reflected in the trajectories of smooth-pursuit of a circle (Fig. 9). This distribution appears to be roughly in agreement with equation (1). Despite the broad meridional distribution of the magnification, our experiments nevertheless demonstrate considerable meridional-specificity, because adaptation was always absent in the, relatively narrow, zero-power axis of the cylinders.

Meridian-specificity of (essentially conjugate) saccadic adaptation was probed more stringently by Deubel (1987). He let subjects and monkeys make saccades, in various directions along various meridians, towards a target that jumped. This design was quite similar to the one adopted by Miller et al. (1981) and Deubel et al. (1986), with the exception that Deubel now examined various meridians. For saccades made along one meridian, he let the target make a second jump, consistently in one direction, during the execution of the saccade. He found that saccades made in that direction, along that specific meridian, gradually changed in size, as a result of this conditioning (adaptation). This direction-specific adaptation transferred, to a lesser extent, to adjacent meridians, with a meridian-specificity on the order of $30 \mathrm{deg}$ to either side. This shows that, at least for binocular saccades, meridional adaptation can be considerably more specific than in our present experiments.

\section{Location of the adaptation in the oculomotor system}

Although our results provide no lower limit of the tuning-width of nonconjugate saccadic adaptation, they show clearly that such adaptation cannot be reduced to a change in magnitude in either the horizontal or vertical saccadic component. At an immediate pre-motor level, separate regions have been identified in the brainstem, which are involved in the generation of horizontal and vertical saccades. Oblique saccades require the concerted action of these regions (Luschei \& Fuchs, 1972; Keller, 1974; Henn \& Cohen, 1976; Büttner, Büttner-Ennever \& Henn, 1977; Hepp \& Henn, 1983; King, Lisberger \& Fuchs, 1986). Therefore, oblique saccades result from the combined generation of their horizontal and vertical components. The way in which a central command to generate an oblique saccade is translated into its horizontal and vertical components, is still subject to debate. Various, somewhat conflicting, models for this decomposition have been proposed (Van Gisbergen, van Opstal \& Schoenmakers, 1985; Tweed \& Vilis, 1985; Fuchs, Kaneko \& Scudder, 1985). None of these, however, can exhaustively account for the various characteristics of oblique saccades (Grossman \& Robinson, 1988).
The important point in the present context is, at what stage of saccade-generation the nonconjugate adaptive changes take place. For the meridian-specific nonconjugate adaptations along the horizontal or vertical meridians, adaptation could have occurred either before or after decomposition of the central motor commands into their horizontal and vertical components. However, if the adaptive changes indeed took place after decomposition, they could only be specific for the horizontal or the vertical meridian, and not for oblique meridians. If, for instance, a size reduction along the $45 \mathrm{deg}$ meridian would be composed of reductions of the vertical, as well as the horizontal component of such saccades, the reduction would have to be similarly expressed in saccades along the $135 \mathrm{deg}$ meridian, and specificity along one oblique meridian with no change along the orthogonal oblique meridian (as was found in our experiments) would be impossible. Therefore, our results show that the plastic changes in the oculomotor commands of obliquely adapted saccades have to take place before these commands are decomposed into their cardinal components, i.e. central to the premotor level. This result confirms Deubel's (1987) conclusion from experiments on conjugate, meridian-specific adaptations to intra-saccadic target-displacements.

\section{Post-saccadic drift}

Post-saccadic drift was adapted asymmetrically along the horizontal meridian only. The direction of this adaptive change was such that it reduced any target-disparities present at saccadic offset. Why these adaptive changes were restricted to the horizontal meridian remains a mystery. As we argued before (Lemij \& Collewijn, 1991a,b), this post-saccadic drift should not be considered as a vergence-movement controlled directly by target-disparity, because the asymmetrically adapted drift was also present during monocular viewing. Obviously, target-disparity is indeterminate with one eye covered.

Although post-saccadic drift has traditionally been viewed as the result of a mismatch between the pulse and the step of saccadic motor commands and therefore as an imperfection in oculomotor control, it might not at all be as imperfect as previously thought. In our experiments, it served a useful purpose by reducing the target-disparities at saccadic offset. In addition, postsaccadic drift can be selectively adapted, without any adaptation of the saccades per se, if the visual world moves steadily and consistently in a specific direction, following a saccade, suggesting that post-saccadic drift serves a specific function in controlling the position of the retinal image (Optican \& Miles, 1985). Furthermore, if one eye is covered for a prolonged period, the characteristics of the post-saccadic drift of the fellow, viewing eye are changed (Kapoula, Hain, Zee \& Robinson, 1987). Taken together, these results suggest that postsaccadic drift does not result from a flaw in saccadic control, but, instead, may represent a deliberate, though not yet fully understood, corrective eye movement, presumably subserving binocular foveation of a target. 


\section{Mechanisms of nonconjugate saccadic adaptation}

A moot point in the discussion of nonconjugate adaptation is its origin in either a reprogramming of saccadic commands as such, or in a conditioned contribution of the vergence system to each saccade. A possible role of the vergence subsystem in the plastic changes of post-saccadic drift and saccades alike would become more likely, if an adaptive linking between saccades and vergence eye movements could be demonstrated. Erkelens et al. (1989) tried to induce nonconjugate, adaptive changes in saccades by having subjects make, for half an hour at a stretch, repetitive gaze-shifts between two continuously present targets that differed both in direction and in distance. Despite this persistent association between version and vergence eye movements, no nonconjugate changes whatsoever occurred. Thus, an adaptive linking between saccades and vergence eye movements has not been demonstrated until now. Oohira et al. (1991) suggested a possible role of the vergence-subsystem in the nonconjugate adaptation of saccades to the wearing of anisometropic spectacles. They argued that the vergence-traces of horizontal saccades, made by a subject who was adapted (by anisometropic spectacles) to make nonconjugate saccades, resembled closely the vergence-traces of horizontal nonconjugate saccades made by the same subject in the unadapted state, between appropriately positioned targets that differed in direction and distance. We feel, however, that their example (shown in their Fig. 10) is far from conclusive, because the vergence-traces in the two experimental conditions were actually quite dissimilar. For vertical adaptations, invoking of the vergence system is even more speculative. Oohira et al. (1991) argued that the difference in the vertical saccades of the two eyes in a subject, adapted to anisometropic spectacles, was reproduced by the same subject in the unadapted state, looking at a horizontally eccentric object, which was closer to one eye than to the other eye, and therefore would also require different vertical saccades. Despite the superficial similarity in the traces of "vertical vergence" (Fig. 11 in Oohira et al., 1991), there are fundamental problems in ascribing this similarity to a robust, disparity-controlled vertical vergence system. Firstly, as the authors note, the "vertical vergence" found with an eccentric object was not present for a midline target seen through the anisometropic spectacles, if no time for adaptation was allowed. Obviously, the vergence system by itself could not cope adequately with the large vertical disparities in the anisometropic condition. Secondly, there is a certain ambiguity in defining, vertical angles subtended by peripheral objects, because these depend on the choice of the coordinate system. In Helmholtz's coordinate system, which has many advantages in describing binocular eye movements (see Carpenter, 1988) and which was used in our present paper, vertical positions are described as the elevation angle of the binocular plane of regard (containing the two lines of sight) with respect to the horizontal plane. Obviously, in this system a normally viewed, physical target causes identical elevations of both eyes, irrespective of its position and relative distance to each of the eyes. Mapped in this coordinate system, the vertical saccades illustrated by Oohira et al. (their Fig. 11, right panel; coordinate system unspecified) would be identical in size, or stated in other words: the intersections of each of the eyes with parasagittal planes through the rotatory centers would still have moved through equal angles in these planes. Therefore, we feel that the interpretation of interocular differences in vertical gaze angles, related to the viewing of eccentric natural targets, as the expression of a vertical vergence system may be fundamentally flawed, especially when the coordinate system conventions used in the recordings are not explicitly discussed. In any case, the situation created by anisometropic spectacles in the vertical dimension can, unlike in the horizontal dimension, not be simulated adequately by the positioning of natural targets.

In conclusion, we feel that at present there is insufficient evidence to decide whether nonconjugate adaptation of saccades is due to a reprogramming of the saccadic commands for each of the eyes apart, or to the close collaboration-and possibly nonlinear interaction-between the saccadic and vergence system. We lean, however, towards an explanation in terms of differential saccadic programming, because this seems more parsimonious. Recalibration of saccades (and other types of eye movements) for a specific plane and eye secms fairly straightforward. To derive such adaptation from a learned association with vergence seems more problematic, because the normal performance of the vergence system seems ill tuned for dealing with vertical and oblique size-differences, and also because the contribution by vergence would have to be opposite in sign for saccades in opposite directions.

\section{REFERENCES}

Abel, L. A., Schmidt, D., Dell'Osso, L. F. \& Daroff, R. B. (1978). Saccadic system plasticity in humans. Annals of Neurology, 4, 313-318.

Büttner, U., Büttner-Ennever, J. A. \& Henn, V. (1977). Vertical eye movement related unit activity in the rostral mesencephalic reticular formation of the alert monkey. Brain Research, 130, 239-252.

Carpenter, R. H. S. (1988). Movements of the eyes (2nd edn). London: Pion Press.

Collewijn, H., Erkelens, C. J. \& Steinman, R. M. (1988a). Binocular co-ordination of human horizontal saccadic eye movements. Journal of Physiology, 404, 157-182.

Collewijn, H., Erkelens, C. J. \& Steinman, R. M. (1988b). Binocular co-ordination of human vertical saccadic eye movements. Journal of Physiology, 404, 183-197.

Collewijn, H., Van der Mark, F. \& Jansen, T. C. (1975). Precise recording of human eye movements. Vision Research, 15, 447-450.

Collewijn, H., Van der Steen, J. \& Steinman, R. M. (1985). Human eye movements associated with blinks and prolonged eyelid closure. Journal of Neurophysiology, 54, 11-27.

Deubel, H. (1987). Adaptivity of gain and direction in oblique saccades. In O'Regan, J. K. \& Lévy-Schoen, A. (Eds), Eye move ments: From physiology to cognition (pp. 181-190). Elsevier: Amsterdam.

Deubel, H., Wolf, W. \& Hauske, G. (1986). Adaptive gain control of saccadic eye movements. Human Neurobiology, 5, 245-253. 
Enright, J. T. (1984). Changes in vergence mediated by saccades. Journal of Physiology, 350, 9-31.

Erkelens, C. J., Collewijn, H. \& Steinman, R. M. (1989). Asymmetrical adaptation of human saccades to anisometropic spectacles. Investigative Ophthalmology and Visual Science, 30, 1132-1145.

Erkelens, C. J., Steinman, R. M. \& Collewijn, H. (1989). Ocular vergence under natural conditions. II. Gaze shifts between real targets differing in distance and direction. Proceedings of the Royal Society of London, Series B, 236, 441-465.

Fuchs, A. F., Kaneko, C. R. S. \& Scudder, C. A. (1985). Brainstem control of saccadic eye movemens. Annual Review of Neuroscience, 8, 307-337.

Grossman, G. E. \& Robinson, D. A. (1988). Ambivalence in modelling oblique saccades. Biological Cybernetics, 58, 13-18.

Henn, V. \& Cohen, B. (1976). Coding of information about rapid eye movements in the pontine reticular formation of alert monkeys. Brain Research, 108, 307-325.

Hepp, K. \& Henn, V. (1983). Spatio-temporal recoding of rapid eye movement signals in the monkey paramedian pontine reticular formation (PPRF). Experimental Brain Research, 52, 105-120.

Hering, E. (1868). Die Lehre vom binokularen, Sehen. Leipzig: Engelman. English translation in Bridgeman, B. \& Stark, L. (Eds), The theory of binocular vision (1977). New York. Plenum Press.

Kapoula, Z., Robinson, D. A. \& Hain, T. C. (1986). Motion of the eye immediately after a saccade. Experimental Brain Research, 61, 386-394.

Kapoula, Z., Hain, T. C., Zee, D. S. \& Robinson, D. A. (1987). Adaptive changes in post-saccadic drift induced by patching one eye. Vision Research, 27, 1299-1307.

Keller, E. L. (1974). Participation of medial pontine reticular formation in eye movement generation in monkey. Journal of Neurophysiology, 37, 316-332.

Kenyon, R. V., Ciuffreda, K. J. \& Stark, L. (1980). Unequal saccades during vergence. American Journal of Optometry and Physiological Optics, 56, 586-594

King, W. M., Lisberger, S. G. \& Fuchs, A. F. (1986). Oblique saccadic eye movements of primates. Journal of Neurophysiology, $56,769-784$.

Kommerell, G., Olivier, D. \& Theopold, H. (1976). Adaptive programming of phasic and tonic components in saccadic eye movements. Investigations in patients with abducens palsy. Investigative Ophthalmology, 15, 657-660.

Lemij, H. G. (1990). Asymmetrical adaptation of human saccades to anisometropic spectacles. Ductoral thesis, Erasmus University, Rotterdam, The Netherlands.

Lemij, H. G. \& Collewijn, H. (1989a). Differences in accuracy of human saccades between stationary and jumping targets. Vision Research, 29, 1737-1748.

Lemij, H. G. \& Collewijn, H. (1989b). Meridian-specific and asymmetrical adaptation of human saccades. European Journal of Neuroscience (Suppl.), 2, 36.
Lemij, H. G. \& Collewijn, H. (1991a). Long-term nonconjugate adaptation of human saccades to anisometropic spectacles. Vision Research, 31, 1939-1954.

Lemij, H. G. \& Collewijn, H. (1991b). Short-term nonconjugate adaptation of human saccades to anisometropic spectacles. Vision Research, 31, 1955-1966.

Luschei, E. S. \& Fuchs, A. F. (1972). Activity of brain stem neurons during eye movements of alert monkeys. Journal of Neurophysiology, 35, 445-461

Miller, J. M., Anstis, T. \& Templeton, W. B. (1981). Saccadic plasticity: Parametric adaptive control by retinal feedback. Journal of Experimental Psychology: Human Perception and Performance, 7, 356-366.

Ogle, K. N. (1962). Special topics in binocular spatial localization. In Davson, H. (Ed.), The eye (Vol. 4, pp. 349 407). New York Academic Press.

Oohira, A., Zee, D. S. \& Guyton, D. L. (1991). Disconjugate adaptation to long-standing, large-amplitude spectacle-corrected anisometropia. Investigative Ophthalmology and Visual Science, 32, 1693-1703.

Optican, L. M. \& Miles, F. A. (1985). Visually induced adaptive changes in primate saccadic oculomotor control signals. Journal of Neurophysiology, 54, 940-958.

Optican, L. M., Zee, D. S. \& Chu, F. C. (1985). Adaptive response to ocular muscle weakness in human pursuit and saccadic eye movements. Journal of Neurophysiology, 54, 110-122.

Robinson, D. A. (1963). A method of measuring eye movements using a scleral search coil in a magnetic field. IEEE Transactions on Biomedical Engineering, BME-10, 137-145.

Schor, C. M., Gleason, G. \& Horner, D. (1990). Selective nonconjugate binocular adaptation of vertical saccades and pursuits. Vision Research, 30, 1827-1844.

Southall, J. P. C. (1961). Introduction to physiological optics. New York: Dover.

Tweed, D. \& Vilis, T. (1985). A two dimensional model for saccade generation. Biological Cybernetics, 52, $219 \cdot 227$.

Van Gisbergen, J. A. M., van Opstal, A. J. \& Schoenmakers, J. J. M. (1985). Experimental test of two models for the generation of oblique saccades. Experimental Brain Research, 57, 321-336.

Zee, D. S. \& Levi, L. (1989). Neurological aspects of vergence eye movements. Revue Neurologique (Paris), 145, 613-620.

Acknowledgements - This research was supported by the foundation for Medical Research MEDIGON (The Netherlands), Grant No. 900-550-092. We thank Drs J. Van der Steen and C. J. Erkelens for their comments and for their aid in writing the necessary computer programs. 